\title{
Schülerperspektiven auf Ergänzungsstunden als Maßnahme individueller Förderung
}

\section{Gruppendiskussionen mit gymnasialen Unter- und Mittelstufenschüler/innen}

\section{David Rott}

Eingegangen: 25. Februar 2020 / Überarbeitet: 7. Dezember 2020 / Angenommen: 10. Dezember 2020 / Online publiziert: 28. Dezember 2020

(C) Der/die Autor(en) 2020

Zusammenfassung Individuelle Förderung gilt als Merkmal guten Unterrichts, wobei individuelle Förderung als Sammelbegriff zu verstehen ist und in bildungswissenschaftlichen, bildungspolitischen und schulpraktischen Auseinandersetzungen unterschiedlich genutzt wird. Grundsätzlich ist darauf zu verweisen, dass individuelle Förderung unterbeforscht ist. Dies gilt sowohl zur Umsetzung individueller Förderung im Allgemeinen wie auch zu konkreten schulbezogenen Maßnahmen im Speziellen. Mit dieser Studie wird die Maßnahme ,Ergänzungsstunden“ (NRW) untersucht. Die ,Ergänzungsstunden“ sind an nordrhein-westfälischen Gymnasien Pflichtstunden, die verstärkt zur individuellen Förderung genutzt werden sollen und an den einzelnen Schulen unterschiedlich eingesetzt werden können. Während die Perspektive der Lehrpersonen auf individuelle Förderung bereits untersucht wurde, liegen Daten von Schüler/innen nur in sehr geringem Maße vor. So ist nicht beforscht, wie Schüler/innen Maßnahmen individueller Förderung wahrnehmen und wie sie diese bewerten. Dies erscheint aber insofern als relevant, als dass Schüler/ innen wichtige Impulse für Schulentwicklungsprozesse unterstützen können, wenn sie in die Evaluation solcher Angebote einbezogen werden. Diese Studie stellt einen ersten Versuch dar, diese Forschungslücke zu schließen. 62 Unter- und Mittelstufen-Schüler/innen (Jahrgangsstufe 6-8) eines Gymnasiums wurden in zehn Gruppendiskussionen zur Umsetzung der ,Ergänzungsstunden“ an ihrer Schule befragt. Die Daten wurden mittels qualitativer Inhaltsanalyse ausgewertet. Die Daten verdeutlichen, dass die Befragten die Angebote individueller Förderung differenziert einschätzen können. Aus den Äußerungen der Schüler/innen lassen sich Impulse für die Schul- und Unterrichtsentwicklung ableiten, aber auch Implikationen für die weitere schulische und wissenschaftliche Praxis beschreiben.

\footnotetext{
D. Rott $(\bowtie)$

Institut für Erziehungswissenschaft, Westfälische Wilhelms-Universität Münster, Bispinghof 5-6, 48143 Münster, Deutschland

E-Mail: david.rott@uni-muenster.de
} 
Schlüsselwörter Individuelle Förderung · Ergänzungsstunden ·

Schülermeinungen · Gruppendiskussion

\title{
Students' perspectives on individual fostering
}

Group discussion concerning 'Ergänzungsstunden'

\begin{abstract}
Individual support is regarded as a major characteristic of good teaching. However, the definition of the term itself as well as the implementation of individual support programs differs in applied educational sciences, educational policy and school practice. Thus, research targets both the advantages and disadvantages of individual support in general as well as the concrete school-related actions in particular. One popular measure is 'Ergänzungsstunden' (NRW), which are additional yet compulsory lessons at North Rhine-Westphalian grammar schools. They should be increasingly used for individual support and can be designed individually at school level. While teachers' perspectives have been investigated repeatedly, this study aims at investigating pupil's perceptions of measures of individual support.

A total of 62 lower and middle school pupils (grades 6-8) of a grammar school took part in ten group discussions about the implementation of 'Ergänzungsstunden' at their school. The data were evaluated using qualitative content analysis. The data show that the respondents are able to make a differentiated assessment of the individual support offered. From the students' statements, impulses for school and lesson development can be derived, but also implications for further school and academic practice can be described.
\end{abstract}

Keywords Individual Fostering · Ergänzungsstunden · Student's Perspective · Group Discussion

\section{Einleitung}

Maßnahmen individueller Förderung gelten als Gestaltungsoption guten Unterrichts (Helmke 2010), wobei Studien verdeutlichen, dass diese im Schulalltag unterschiedlich konzipiert und durchgeführt werden (Rott 2017). Zudem sind Maßnahmen individueller Förderung aufgrund ihrer Pluralität empirisch schwer zu fassen (Corno 2008; Fischer 2014; van Geel et al. 2019). Deutlich wird, dass der bildungspolitische und -wissenschaftliche Diskurs in Deutschland nicht klar voneinander getrennt sind und in den Bildungswissenschaften bildungspolitische Argumentationsmuster übernommen werden (Klieme und Warwas 2011). Dies wird vielfach auch kritisch betrachtet (Bellmann 2016; Trautmann und Wischer 2011).

Ein Aspekt, der in der Debatte um Maßnahmen individueller Förderung bislang weitestgehend unbearbeitet ist, ist die Frage, wie Schüler/innen diese wahrnehmen. Schüler/innen sind zentrale Akteur/innen des Unterrichtsgeschehens und nehmen ihren Unterricht anders wahr, als dies Lehrpersonen oder Eltern tun. Anders als im Kontext von Inklusion, wo Perspektiven von Schüler/innen auf gemeinsames Lernen mit unterschiedlichen Perspektiven auf konkrete fachliche Rahmungen (Obrusnikova et al. 2010) bzw. Formen der Beeinträchtigungen (Bosse et al. 2018) abzielen, 
liegen für Maßnahmen individueller Förderung sowohl national als auch international keine Studienergebnisse vor. Dabei scheinen die Einschätzungen von Schüler/ innen insgesamt von großer Bedeutung zu sein. Deutlich wird dies in der Relevanz, die dem Feedback von Schüler/innen an Lehrpersonen zugeschrieben wird: Gruehn (2000) zeigt, wie Schülermeinungen für Fragen von Schul- und Unterrichtsentwicklung mit eingebunden werden können. Hattie (2014) verweist auf den hohen Effekt, den das Feedback von Schüler/innen an Lehrpersonen mit Blick auf die kognitive Anregung im Unterricht geben kann. Dieser Spur, die Einschätzungen von Schüler/ innen für die Untersuchung von Maßnahmen individueller Förderung fruchtbar zu machen, soll in diesem Artikel weiter nachgegangen werden.

Durch bildungspolitische Vorgaben werden zudem Maßnahmen individueller Förderung zunehmend an Schulen herangetragen. So etwa auch an die Gymnasien in Nordrhein-Westfalen (NRW). In NRW ist mit den sogenannten Ergänzungsstunden (Schulministerium NRW [MSB], o.J.) eine organisatorische Perspektive geschaffen worden, mit der an Gymnasien Zusatzangebote individueller Förderung umgesetzt werden können. Im Wechsel von G8 auf G9 werden so Mittel in Form von Unterrichtsstunden explizit für individuelle Förderung vergeben. Studien zu den Ergänzungsstunden liegen bislang allerdings noch nicht vor.

An diesem doppelten Desiderat setzt der Beitrag an. Vor dem Hintergrund der skizzierten Problemlage werden zunächst Überlegungen zu Umsetzungsformen individueller Förderung und das Konzept der nordrhein-westfälischen Ergänzungsstunden aufgearbeitet. Hieraus wird die leitende Fragestellung der Studie abgeleitet: Wie nehmen Schüler/innen Maßnahmen individueller Förderung in den Ergänzungsstunden wahr? Um herausarbeiten zu können, welche Perspektiven Schüler/innen bezogen auf individuelle Förderung einnehmen, wurden an einem Gymnasium 62 Schüler/innen in zehn Gruppendiskussionen befragt. Die Daten wurden mittels inhaltlich strukturierender qualitativer Inhaltsanalyse (Kuckartz 2016) ausgewertet.

\section{Umsetzungsformen individueller Förderung}

Individuelle Förderung als Begriff, der bildungspolitisch, schulpädagogisch und bildungswissenschaftlich in differenzierter Weise genutzt wird, wird in der Forschungsperspektive äußerst kritisch angefragt (Hasselhorn et al. 2018), da Bildung im Allgemeinen und Lernen im Speziellen im Sinne konstruktivistischer Verständnisse immer individuell zu denken sind. Dennoch hat sich der Begriff seit den 1970erJahren (etwa Nunner-Winkler 1971) langfristig etabliert und mit der Veröffentlichung der ersten PISA-Studie noch einmal an Diskurskraft gewonnen (Trautmann und Wischer 2011).

Es liegt eine Vielzahl von Definitionen zur individuellen Förderung vor (Rott 2017, S. 28 ff.), wobei Kunzes (2010) Definition als eine gewertet werden kann, die im bildungswissenschaftlichen Diskurs breit rezipiert und auch akzeptiert ist. Kunze (2010, S. 19) definiert individuelle Förderung als ,alle Handlungen von Lehrerinnen und Lehrern und von Schülerinnen und Schülern [...], die mit der Intention erfolgen bzw. die Wirkung haben, das Lernen der einzelnen Schülerin/des einzelnen Schülers unter Berücksichtigung ihrer/seiner spezifischen Lernvoraussetzungen, -bedürfnisse, 
-wege, -ziele und -möglichkeiten zu unterstützen“. Diese Definition geht von der direkten Interaktion zwischen Lehrpersonen und Schüler/innen aus. Wird versucht, den Begriff weniger auf die konkrete pädagogische Ausgestaltung von Schule und Unterricht zu beziehen als vielmehr auf eine systemische Betrachtung hinzuwirken, bieten sich andere Strukturierungsversuche an.

Mit Blick auf die dem Begriff innenliegende Komplexität verweist Fischer (2014, S. 32) darauf, dass individuelle Förderung ein Sammelbegriff ist, ,dem sich verschiedene Ansätze und bestehende Konzepte zuordnen lassen, beispielsweise Differenzierung und Individualisierung, offener und adaptiver Unterricht sowie Forderund Förderunterricht“". Individuelle Förderung ist demnach ein Konzept, das aus verschiedenen Warten heraus in der pädagogischen und wissenschaftlichen Praxis bearbeitet werden kann. Hierin liegt wiederum ein zentrales Problem, auf das Klieme und Warwas (2011) verweisen: Individuelle Förderung ist ein vergleichsweise unspezifisches Konzept, es hat keine festen Grenzen und ist von daher schwer untersuchbar.

Mit Hess und Lipowsky (2017) lässt sich argumentieren, dass Anstrengungen hin zur individuellen Förderung im Unterricht oftmals lediglich die Ausweitung des Methodenrepertoires, die Nutzung verschiedener Sozialformen oder aber übergreifender Organisationsformen betreffen. Aktuelle Studien vergegenwärtigen diese Perspektivvielfalt. Lingemann (2017) greift auf eine Studie von Solzbacher (2016) zurück und stellt heraus, dass Maßnahmen individueller Förderung die Bestrebungen der Schulentwicklung insgesamt betreffen und ausgehend von den Prämissen des Kollegiums zu gestalten sind. Individuelle Förderung wird als Maßnahmenpaket verstanden, das eine pädagogische Aufgabe beschreibt. Für das Gymnasium liegen bislang nur wenige themenbezogene Studien vor, wobei oft Einzelprojekte (Kühn 2014) oder Berichte aus Forschungsprojekten (Lindemann et al. 2014) vorgestellt werden.

Perspektiven von Schüler/innen auf Maßnahmen individueller Förderung werden in den gegenwärtigen Studien nicht erhoben. In aktuellen Studien werden Schülerperspektiven auf inklusiven Unterricht erfasst (Bosse et al. 2018), die verdeutlichen, wie Schüler/innen Settings gemeinsamen Lernens wahrnehmen. In diesen Studien wird die Relevanz der Schülerperspektiven deutlich: Sie können Unterricht bzw. die Gegebenheiten, in denen Unterricht stattfindet, differenziert wahrnehmen und beschreiben. Dies gilt sowohl für Schüler/innen der Grundschule als auch der weiterführenden Schulen. Abgehoben wird zumeist auf die Einbindung von Schüler/innen mit und ohne Beeinträchtigungen in den Unterricht und die Wahrnehmung dieser Differenzen. Konkrete Bezüge zum Unterrichtsgeschehen, etwa zur Unterrichtsqualität, werden nicht hergestellt. Mit Blick auf Maßnahmen individueller Förderung ergibt sich hier ein entscheidendes Forschungsdesiderat. Wird individuelle Förderung als Gesamtunterrichtsstrategie verstanden, ist auf ein umfangreiches Wahrnehmen des Unterrichts abzuheben. Wie andere Forschungszusammenhänge zeigen, können Schüler/innen durch ihre Einbindung in den Unterricht ein differenziertes Bild der Ausgestaltung pädagogischer Situationen leisten. Wird, wie in dieser Studie angestrebt, eine konkrete einzelne Maßnahme untersucht, lässt sich eine Schärfung der Perspektive auf den Unterricht im Gesamten anstreben. Durch die Beschreibung kon- 
kreter Einzelmaßnahmen wird eine strukturelle Differenz zum regulären Unterricht beschreibbar gemacht.

\section{Ergänzungsstunden als bildungspolitische Maßnahme individueller Förderung}

Die Ergänzungsstunden in NRW sind ein Programm für Gymnasien, das dazu beitragen soll, mangelnde Schulleistungen zu kompensieren, um ein drohendes Sitzenbleiben oder einen Schulwechsel zu verhindern. Anknüpfungspunkt ist ,,vorrangig [...] die Intensivierung der individuellen Förderung der Kompetenzen in Deutsch, Mathematik, den Fremdsprachen oder in den Naturwissenschaften“ (MSB o.J.). Zusätzlich können Schulen die Ergänzungsstunden nutzen, um die Profilbildung voranzutreiben. Die Ergänzungsstunden umfassen ,am Gymnasium mit neunjährigem Bildungsgang acht, am Gymnasium mit achtjährigem Bildungsgang fünf Stunden“ (a.a.O.) und sind „nicht für alle Schülerinnen und Schüler verpflichtend“ (a.a.O.). Die Schulen können demnach entscheiden, für welche Schüler/innen sie Angebote im Rahmen der Ergänzungsstunden bereithalten. Der kompensatorische Charakter ist in den Ausführungen des Schulministeriums tonangebend. Gleichzeitig sollen die Ergänzungsstunden dazu beitragen, die schuleigenen Stärken in entsprechenden Profilen auszudifferenzieren. Für diese Aufgabe werden den Schulen Ressourcen in Form von Unterrichtsstunden bereitgestellt. Ergänzungsstunden stellen damit einen bildungspolitisch finanzierten Rahmen für die explizite Durchführung individueller Förderung dar. Die Ausgestaltung dieser Formate ist jeder Schule selbst überlassen. Inwiefern unter dem Label Ergänzungsstunden Maßnahmen der individuellen Förderung tatsächlich stattfinden, ist mit dieser Ausrichtung demnach nicht geklärt.

Jede Schule muss für die Ergänzungsstunden ein Konzept erstellen. Eine erste Recherche zeigt, dass die Schulen die Ergänzungsstunden unterschiedlich in den pädagogischen Alltag einbetten. Eine tiefergehende Analyse der Konzepte steht allerdings noch aus. Um einen analytischen Einblick in Umsetzungsformen der Ergänzungsstunden zu erhalten, wurde die hier vorzustellende Studie durchgeführt.

\section{Schülerperspektiven auf Ergänzungsstunden am Beispiel der Lumosstunden}

Die Einsatzmöglichkeiten der Ergänzungsstunden werden exemplarisch anhand eines Gymnasiums ${ }^{1}$ einer Kleinstadt im Münsterland vorgestellt. Für die Ergänzungsstunden wurde das schuleigene Konzept Lumosstunden entwickelt, das in der fünften Jahrgangsstufe einsetzt und in verändernder Form in den höheren Jahrgangsstufen weitergeführt wird. Die Stunden sind so konzipiert, dass sie die Woche rahmen: Montags beginnt die Woche mit zwei Lumosstunden, Freitagmittag endet sie entspre-

\footnotetext{
1 Um die Anonymität der Schule sicherzustellen, wurden alle Daten, etwa Projektnamen, verändert. Die Informationen zur Umsetzung der Ergänzungsstunden stammen aus Schuldokumenten sowie Gesprächen mit verantwortlichen Personen. Diese werden aus Gründen der Anonymisierung nicht weiter angegeben.
} 
chend. Die Stunden werden geleitet von Fachlehrpersonen in Mathematik, Deutsch bzw. Englisch. Alle Schüler/innen der Schule der Klassen 5-8 nehmen an den Lumosstunden teil. Damit bietet die Schule die Ergänzungsstunden nicht nur für einige Schüler/innen an, sondern weitet sie auf alle Schüler/innen aus.

In den Lumosstunden der fünften Klasse erhalten die Schüler/innen Übungsaufgaben inklusive Lösungsblätter in den Hauptfächern von den Fachlehrpersonen der jeweiligen Klasse. Die Schüler/innen sollen ihre Aufgaben eigenständig auswählen und kontrollieren und dabei alle drei Fächer berücksichtigen. Nach Erledigung der Aufgaben können Schüler/innen in Projektform Arbeiten oder Präsentationen zu frei gewählten Themen erstellen. Alle dürfen das Klassenzimmer verlassen und sich einen eigenen Platz suchen. Hinzu kommen weitere Projekte, die von allen Schüler/ innen gemeinsam bearbeitet werden, etwa zur Stärkung der Sozialkompetenz, der Verbesserung der Lern- und Arbeitsstrategien oder zur Gesundheitsförderung. Damit sind die Lumosstunden zunächst als kompensatorische Maßnahme zu verstehen, die sich durch Übungs- und Wiederholungsaufgaben auszeichnen. Gleichzeitig findet eine Öffnung dieser Strukturen statt, indem die Schüler/innen entscheiden können, in welcher Reihenfolge, an welchem Ort oder in welcher Sozialform sie die Aufgaben bearbeiten wollen.

In der sechsten Klasse werden die Lumosstunden vergleichbar fortgesetzt. Hier bleibt der kompensatorische bzw. auch angleichende Charakter erhalten. Ab der siebten Klasse werden sie in den Nachmittagsunterricht integriert: Fachspezifische, klassenübergreifende Lerngruppen (etwa Mathematik, Latein) werden eingerichtet und von einer Fachlehrperson geleitet. Die Angebote finden also nicht mehr im Klassenverband statt. Für Schüler/innen, die in den Hauptfächern mindestens gut stehen, entfallen die Übungsangebote. Sie besuchen Interessenskurse, in denen sie ein Themengebiet kennenlernen. Hierzu zählen kulturelle (etwa Theater), sportliche, sprachliche oder MINT-Angebote (etwa Programmieren). Diese Kurse werden für ein Trimester belegt, danach erfolgt eine Umverteilung: Neue Kurse können ausprobiert, bestehende weitergeführt werden. Je nach Leistungsentwicklung müssen Übungskurse angewählt oder verlassen werden. Die Teilnahme an den Kursen wird auf dem Zeugnis vermerkt. Eine Benotung findet nicht statt.

Die Lumosstunden in der siebten Jahrgangsstufe erhalten damit ihren kompensatorischen Charakter, geben aber auch mehr Freiheiten für diejenigen, die gute oder sehr gute Leistungen in den sogenannten Kernfächern erbringen. Im Sinne der bildungspolitischen Vorgaben findet eine Profilschärfung der Schule statt. Erziehungswissenschaftlich reflektiert ist zu erkennen, dass eine interessensorientierte Förderung dann ermöglicht wird, wenn die als grundlegend eingestuften Leistungen in den Hauptfächern erbracht wurden. Die Öffnung des Unterrichts erfolgt demnach durch äußere Differenzierung (Zuteilung auf verschiedene Angebote), wobei weitere Öffnungsformen (Wahl des Gegenstandes, des Materials oder der Sozialform) stark in den jeweiligen Jahrgängen und Angeboten variieren. 


\section{Fragestellung und Annahmen}

Ausgehend von den theoretischen Vorüberlegungen und den organisatorischen Gegebenheiten in den Lumosstunden als ein Beispiel für die Umsetzung der Ergänzungsstunden in NRW wurde folgende Forschungsfrage aufgestellt: Wie nehmen Schüler/ innen Maßnahmen individueller Förderung in den Ergänzungsstunden wahr?

Damit einher geht die Annahme, dass die Schüler/innen die Lumosstunden anders wahrnehmen als den regulären Unterricht. Die verschiedenen Erfahrungen der Schüler/innen in den Lumosstunden lassen vermuten, dass die Befragten auf unterschiedliche Aspekte in ihren Argumentationen Bezug nehmen. Im Sinne des Konzeptes der Lumosstunden wäre davon auszugehen, dass die Schüler/innen die Ergänzungsstunden als kompensatorische Maßnahme wahrnehmen, durch die sie ihre schulischen Leistungen verbessern können. Die geöffnete Struktur der Lumosstunden lässt die Vermutung zu, dass die Schüler/innen sich in den Lumosstunden freier erleben als im regulären Unterricht.

\section{Forschungsmethodisches Vorgehen}

Um die Forschungsfrage Wie nehmen Schüler/innen Maßnahmen individueller Förderung in den Ergänzungsstunden wahr? beantworten zu können, wurden Gruppendiskussionen mit Schüler/innen durchgeführt. Die Gruppendiskussion ist eine Methode, ,die Daten durch die Interaktion der Gruppenmitglieder gewinnt“ (Mäder 2013, S. 25). „Gruppendiskussionen mit Kindern sind eher selten“ (Heinzel 2013, S. 711); gleiches gilt für Gruppendiskussionen mit Jugendlichen. Dies liegt daran, dass Kindern (und Jugendlichen) unterstellt wird, sie würden sich nicht so äußern, wie dies die Methode vorsieht. Heinzel (ebd.) weist darauf hin, dass durch Gruppendiskussionen Kinder in ihrer Meinungsäußerung bestärkt werden können, da sich das Machtgefälle im Gegensatz zum Interview ändert und die Erwachsenen weniger präsent sind als die Interviewten.

Im Gegensatz zu Interviews können Gruppendiskussionen ein tieferes Verständnis über das Gesagte erzeugen, da die Sprechenden miteinander in Interaktion stehen und Gemeinsamkeiten und Unterschiede verdeutlichen können (Flores und Alonso 1995). Es lässt sich vermuten, dass dies auch für Unter- und Mittelstufenschüler/ innen gilt. Genutzt werden zwei zentrale gruppendiskussionsspezifische Aspekte: Erstens gibt es ein gesetztes Thema und zweitens haben alle Teilnehmenden einen Bezug zu dieser Thematik.

Durch das Gespräch in der Gruppe ist es möglich, informelle Gruppenmeinungen zu erschließen und gemeinsame Orientierungsperspektiven aufzudecken (Mäder 2013, S. 27 f.). Das Aushandeln des Themas durch die Teilnehmenden (Flores und Alonso 1995) legt die heterogenen Einstellungen, Bewertungen und Orientierungsmuster bezogen auf die Lumosstunden offen und bietet die Chance, diese vertiefend zu verstehen und darzustellen zu können. 


\subsection{Stichprobe}

Die Studie wurde im Schuljahr 2017-18 in den Jahrgangsstufen 6-8 durchgeführt. Damit wurden Schüler/innen eingebunden, die selbst Erfahrung mit dem Lumosstunden-Konzept haben. Die Schüler/innen der Jahrgangsstufen 6 und 7 kennen das Konzept seit der fünften Klasse. Die Achtklässler/innen haben ein Vorläufermodell kennengelernt und dieses in den Klassen 5-7 durchlaufen. In der 8. Jahrgangsstufe gibt es für sie zum Befragungszeitpunkt kein entsprechendes Angebot.

67 Schüler/innen sollten für Gruppendiskussionen aus allen zwölf Klassen (vier pro Jahrgang) gewonnen werden. Dafür wurde ein randomisiertes Verfahren eingesetzt, um sicherzustellen, eine möglichst breite Streuung unter den Schüler/innen zu erreichen, ohne auf die Nominierung durch Lehrpersonen angewiesen zu sein. Letztendlich nahmen 62 Schüler/innen (29 Jungen, 33 Mädchen) aus zwölf Klassen der Jahrgangsstufen 6-8 (jeweils vierzügig) teil.

Durchgeführt wurden zehn Gruppendiskussionen mit sechs (acht Gruppendiskussionen) bzw. sieben Schüler/innen (zwei Gruppendiskussionen). In den Gruppendiskussionen wurden die Schüler/innen klassen- und jahrgangsübergreifend gemischt, wobei sich jeweils mindestens zwei Schüler/innen aus der Klasse kannten.

Damit sollte erreicht werden, dass die Schüler/innen zum einen einen gemeinsamen Erfahrungshorizont haben, zum anderen gezwungen sind, die Argumente auszuschärfen, da sich die Erfahrungen unterscheiden. Erreicht werden sollte eine Dynamik, die tiefe Einblicke in Argumentationsstrukturen der Teilnehmenden ermöglicht (Flick 2012, S. 248). Die Gruppendiskussionen wurden an der Schule in einem Raum der Schülervertretung und in einem Konferenzraum an einem Vormittag durch den Autor der Studie sowie eine studentische Mitarbeiterin durchgeführt. Die Dauer lag zwischen 19 und $44 \min (\bar{X}=27,4 \mathrm{~min})$.

\subsection{Leitfaden und Datenauswertung}

Der Leitfaden für die Gruppendiskussion zielte auf ein möglichst selbstlaufendes Gespräch ab. Als Einstieg wurden die Schüler/innen begrüßt, die Vertraulichkeit der Daten erklärt und die Freiwilligkeit der Teilnahme betont. Da die Schüler/innen sich untereinander nicht unbedingt kannten, gab es eine Vorstellungsrunde. Gegeben wurde ein erzählauffordernder Einstieg: Ihr alle nehmt in euren Klassen an den Lumosstunden teil. Mich interessiert, wie ihr das Angebot findet. Erzählt mal, was ihr in den Lumosstunden so macht. Hinzugezogen werden konnten Nachfragen (etwa: Was findet ihr an den Lumosstunden besonders gut/schlecht? Wie findet ihr die Materialien?).

Die Gruppendiskussionen wurden mittels Diktiergerät aufgenommen, die Audiodatei archiviert und mit Hilfe des Computerprogramms f4 nach den Regeln der literarischen Umschrift transkribiert (Gläser-Zikuda 2011, S. 111) und ausgewertet. Die Datenanalyse erfolgte anhand der Kriterien der inhaltlich strukturierenden qualitativen Inhaltsanalyse (Kuckartz 2016) sowohl deduktiv als auch induktiv. Das Material wurde intensiv gelesen und kategorisiert. Für die Analyse wurde das Programm MaxQDA genutzt. Dies ermöglichte einen strukturierenden Zugang zum Material und eine Sortierung anhand der Kategorien. Im Laufe der Analyse wurden 
die Kategorien mit Beispielen versehen und schrittweise ausgeschärft. Die Datenanalyse erfolgte durch den Autor der Studie, wobei die Kategorien in einer kollegialen Beratung auf Problemstellen geprüft und weiterentwickelt wurden. Zudem wurde konsensuell codiert (Kuckartz 2016), das heißt, Problemstellen wurden gemeinsam mit einer Mitarbeiterin besprochen, um ein nachvollziehbares Kategoriensystem erstellen zu können.

\section{Ergebnisse}

In dem Analyseprozess wurden die Kategorien induktiv und deduktiv entwickelt. Deduktiv wurde an das Material herangetragen, dass Maßnahmen individueller Förderung nicht einem starren Muster bzw. Programm folgen, sondern in der pädagogischen Praxis unterschiedlich umgesetzt werden. Entsprechend wurde deduktiv die Kategorie Inhaltliche Gestaltung gebildet, um einen Einblick in ebendiese Umsetzung in den Lumosstunden erhalten zu können. Da die Lumosstunden als Ergänzungsstunden ein Additiv zum regulären Unterricht darstellen, gleichzeitig aber in den Stundenplan eingebunden sind, wurde die Differenz zum regulären Unterricht als Kategorie vorab aufgestellt. Induktiv ließen sich aus dem Material zusätzlich folgende Hauptkategorien entwickeln: Rollen der Zuständigkeiten sowie die Lernatmosphäre in den Ergänzungsstunden. Insgesamt wurden in den vier Hauptkategorien 324 Codings gesetzt. Die Ergebnisdarstellung erfolgt anhand der Hauptkategorien, wobei die Subkategorien helfen, eine differenzierte Betrachtung der Argumentationsmuster der Schüler/innen offenzulegen.

\subsection{Inhaltliche Gestaltung}

Zu der Kategorie Inhaltliche Gestaltung werden Codings gezählt, wenn sie sich auf die Durchführung der Lumosstunden beziehen. Gesetzt wurden insgesamt 109 Codings. Hierzu zählen Aufgaben (45 Codings), fachunspezifische Tätigkeiten (27 Codings), die sich nicht auf ein konkretes Unterrichtsfach beziehen, Projekte (30 Codings) und die Gewichtung der Fächeranteile (7 Codings).

\subsubsection{Aufgaben}

Codings wurden der Kategorie ,Aufgaben“ zugeordnet, wenn die Schüler/innen sich explizit auf die Gestaltung der gegebenen Aufgaben in den Lumosstunden beziehen. Hierzu zählen auch Aussagen zur Bearbeitungszeit oder zum eingesetzten Material, das die Schüler/innen bearbeiten sollen. In allen zehn Gruppendiskussionen wurden die Aufgaben als Thema gesetzt.

Bei den Aufgaben schätzen die Schüler/innen unterschiedlich ein, ob die Bearbeitungszeit ausreichend ist: „Also ich fande dass die Aufgabenpakete manchmal ziemlich gro $\beta$ waren, [...] u:nd ich fand das war dann schon ziemlich viel“. Während einige die Zeit zu knapp bemessen finden, nehmen andere Schüler/innen durchaus Leerlauf wahr. 
Das Übungsmaterial wird als umfangreich beschrieben: „In Mathe [...] ham wir auch immer solche Ordner gehabt, wo man die man dann durcharbeiten (.) sollte oder konnte und die warn auch recht viele so, dass man dann obwohl man das vielleicht konnte, sollte man halt welche davon machen, und dann konnte man sich halt auf die Sachen die man noch nich so gut konnte, nich so ganz konzentrieren“. Kritisiert wird, dass nicht nur an Problemen gearbeitet, sondern ein allgemeines Üben verlangt wird. Gewünscht wird eine genauere Passung zwischen den Entwicklungsbereichen und den Übungsaufgaben.

Die Möglichkeit, eigene Prioritäten festzusetzen, wird positiv bewertet, wenn sie erlebt wird: „Also mir hat gut gefallen, dass wir halt uns selbst aussuchen durften, was wir für $n$ Thema machen, also ob wir jetzt Mathe Englisch oder Deutsch machen, und man halt auch das Fach aussuchen konnte, in dem man nicht so gut war". Andere Schüler/innen erleben die Lumosstunden deutlich gerichteter, sie erfahren eher eingeschränkte Wahlmöglichkeiten, wobei die Varianz auch durch die Lerngruppenzugehörigkeit zu erklären ist. Dies gilt auch für die Qualität der Übungsaufgaben.

Der Faktor des Gewinnens von Sicherheit, etwa bezogen auf Klassenarbeitsvorbereitungen, wird von den Schüler/innen hervorgehoben. Auch leistungsstarke Schüler/innen schätzen es als vorteilhaft ein, Übungsaufgaben zu erhalten: ,Ja also ich fand besonders gut, dass wenn man irgendwie sich in einem Fach noch nicht so ganz sicher fühlt, also dass man dann die Aufgaben zu diesem bestimmten Fach dann machen konnte damit man dann zum Beispiel beim nächsten Mal (irgendwie) ne bessere Note schreibt".

\subsubsection{Fachunspezifische Tätigkeiten}

Als ,Fachunspezifische Tätigkeiten“ wurden Aussagen dann codiert, wenn die Schüler/innen Handlungen beschreiben, die nicht direkt mit einer gestellten Aufgabe oder einem der Unterrichtsfächer Mathematik, Deutsch oder Englisch zu verbinden sind. Diese Kategorie ist damit als Differenz zur Kategorie ,Aufgabe“ zu benennen. In allen zehn Gruppendiskussionen wurden fachunspezifische Tätigkeiten thematisiert.

In den Lumosstunden, ,lernen [wir] dann halt auch irgendwas über unser weiteres Leben", es geht also auch um weiterführende Fragen, die die individuellen Entwicklungen betreffen können oder die Klassengemeinschaft besonders ansprechen. Ein zentraler Aspekt ist das ,Lernen lernen “ und das Zusammenkommen der Fünftklässler/innen zu Beginn der Gymnasialzeit. Hier wird den Lumosstunden eine besondere Rolle zugewiesen: „Und dass man vielleicht [...] die Fünfer oder sowas da irgendwie denen nochmal zeigt weil die wissen vielleicht ja gar nicht wie man richtig lernt oder sowas, weil ich hab jetzt in der Grundschule auch nicht gelernt, und dass man das denen eben zeigt wie man überhaupt richtig lernt".

Nach dem Erledigen der Pflichtaufgaben nutzen die Schüler/innen die Freiräume auch für Spiele und Sprechen. Hierzu werden vor allem Räume außerhalb der Klasse aufgesucht. Dies wird durchaus als wichtig eingeordnet: „Ich fand's sehr gut, dass man diese Selbstständigkeit hatte [...] @ also natürlich wurden die Lumosstunden nicht immer nur zum Lernen genutzt, @ sondern auch zum Diskutieren auch, also von (.) uns Schülern her, aber ich fand das eigentlich sehr gut, dass wir diese (.) Selbstständigkeit sehr gut nutzen konnten“. 


\subsubsection{Projekte}

In allen zehn Gruppendiskussionen wurden die Projekte als Thema gesetzt. Die Projekte, die die Schüler/innen in den Klassen 5 und 6 absolvieren können, wenn sie ihre Übungsaufgaben erledigt haben, finden bei den Schüler/innen generell großen Anklang. Die Leistungsstarken erleben diese als Raum, in dem sie ihre Interessen verfolgen und vertiefen können. Dass sich die Produkte unterscheiden (Texte, Präsentationen, Plakate), wird als flexibel wahrgenommen. Es besteht eine starke Kopplung zwischen Arbeitsgeschwindigkeit und Leistungserbringung in Prüfungssituationen und der Möglichkeit, eigene Projekte zu gestalten. Die Struktur wird durch Ordner gewährleistet, in denen Aufgaben und Arbeitsschritte definiert sind. „Das war halt auch irgendwie bei jedem so gleich, nur dass es dann einmal n anderes Thema war, und dann hat am Ende eigentlich sozusagen (.) das Gleiche gemacht". Einige Schüler/innen nehmen aber auch im vermeintlich offenen Format Begrenzungen wahr.

\subsubsection{Gewichtung der Fächer}

Durch die Möglichkeit, die fachlichen Inhalte in unterschiedlicher Reihenfolge oder Gewichtung zu bearbeiten, erhalten die Schüler/innen Autonomie und Selbstverantwortung für das eigene Handeln. ,Also eigentlich waren die Lumosstunden (.) ganz angenehm, weil man dann halt (.) sich aussuchen konnte, ob man Deutsch Englisch oder Mathe macht, und es war auch so abwechselnd, man konnte sein eigenen Lernrhythmus machen und nich so wie in normalen Unterrichtsstunden, dass man da einfach (.) das macht was der Lehrer sagt, sondern das kann man sich halt einteilen". In vier der zehn Gruppendiskussionen wurde dieses Thema aufgegriffen.

Gewünscht wird eine Erweiterung der Lumosstunden über die Kernfächer hinaus. Dabei spielt der Leistungsstand eine Rolle, um Zeit zu bekommen, seine Schwierigkeiten systematisch zu bearbeiten. Die Idee wird gegeben, ,dass die dann halt in Sport $n$ bisschen Hilfe kriegen, oder die in Kunst und Musik nicht gut sind, dass die dann Hilfe in Kunst und Musik kriegen, oder halt in anderen Nebenfächern zum Beispiel Geschichte, oder (.) jetzt Chemie, Bio“.

Berichtet wird, dass die fachbezogenen Aufgaben stark variieren können und dass eine Absprache zwischen den Lehrpersonen nicht immer gegeben zu sein scheint. Es wird diskutiert, dass wochenweise einzelne Fächer besonders stark mit Material ausgestattet werden, andere aber keine weiteren Materialien bekommen. Dies wird von den Schüler/innen als Ungleichgewicht erlebt.

\subsection{Rollen und Zuständigkeiten}

Die Lehrpersonen werden in ihrer Rolle in den Lumosstunden unterschiedlich beschrieben. 56 Textstellen wurden für diesen Kontext codiert. Die Schüler/innen berichten, dass die Fachlehrpersonen besonders ihr eigenes Fach im Blick haben und darauf pochen, dass diese Aufgaben erledigt werden. Dies wird aber nicht nur negativ konnotiert, sondern auch positiv herausgestellt: „Die war auch unsere Mathelehrerin und das war dann praktisch, weil wir dann auch meistens dann mehr Mathe gemacht 
haben, [...] und dann konnte die uns das direkt erklären“. Das Verhalten der Lehrpersonen wird als ,n bisschen lockerer" beschrieben, ,weil im Unterricht da wollen die natürlich die ganze Zeit irgendwas beibringen, (.) ja und in den Lumosstunden (.) ja da irgendwie nicht ganz so".

Die Nutzung der Räume, das freie Bewegen im Schulgebäude, wird zumeist positiv beschrieben. Allerdings sind die Lehrpersonen bei Konflikten nicht unbedingt greifbar: „Obwohl manchmal gab's auch Streit, als die Lehrer dann unten warn bei den anderen in der Bibliothek [...] und dann (.) ist es halt auch manchmal dazu gekommen dass diese Kästen dann umgeschmissen wurden, und dann fehlten halt irgendwie die Lösungen".

Das offene Arbeiten wird auch von den Schüler/innen als Herausforderung gesehen und die Lehrpersonen werden in ihrem Verhalten gewürdigt: „Also ich fand dass die Lehrerin sich da viel Mühe mit gegeben hat, die hat auch so ne Übersicht sag ich mal gemacht, wo man's dann abhaken sollte, und dann musste $n$ Lehrer das dann auch nachgucken". In allen zehn Gruppendiskussionen wird auf die Rolle der Lehrpersonen Bezug genommen, während nur in zwei Gruppendiskussionen explizit auf die Rolle der Schüler/innen abgehoben wird.

\subsection{Bezüge zum regulären Unterricht}

Die Bezüge zum Regelunterricht werden in allen Gruppendiskussionen deutlich und an 28 Textstellen codiert. Sie werden von den Schüler/innen vor allem in einer verbindenden Weise gezogen, etwa wenn es darum geht, nicht verstandene Inhalte nachzuarbeiten und Inhalte zu wiederholen: ,Also ich fand's ganz gut und praktisch, weil man nochmal den Stoff nachholen konnte, den man im Unterricht durchgenommen hat". Die Lage der Lumosstunden zu Beginn um zum Ende der Woche wird im Gesamtgefüge als sinnvoll erachtet: „Ja und die sind eben halt so ganz gut [...] weil man dann eben nochmal in die in die Woche so reinkommt und die beendet so mit was man [...] durchgenommen hat". Die Lumosstunden haben demnach eine moderierende und einordnende Funktion und geben Orientierung.

Gewünscht wird von den Schüler/innen eine Erweiterung der Angebote (Übungen, Material, etc.) über das Angebot des regulären Unterrichts hinaus: „Bei uns waren sehr häufig die Lehrer nicht da, weswegen man bei Fragen auch nich wirklich ne Antwort bekommen hat, und die Lernmaterialien waren eigentlich genau dieselben wie im Unterricht und meistens hat man das schon im Unterricht verstanden; und hat dann halt in den Lumosstunden gar nichts gemacht". Eingefordert wird von den Schüler/innen, dass die Lehrpersonen ein Angebot gestalten, das Anknüpfungspunkte ermöglicht, aber das Nichtverstehen im Unterricht berücksichtigt und Wiederholungen, Vertiefungen oder Ergänzungen gezielt vorgenommen werden. An dieser Stelle gehen die Einschätzungen der Schüler/innen über reines Üben hinaus.

\subsection{Lernatmosphäre}

Die Lernatmosphäre wird von den Schüler/innen differenziert beschrieben und in sieben der zehn Gruppendiskussionen herausgestellt. Insgesamt 18 Codings wurden zu diesem Themenschwerpunkt herausgearbeitet. Zum einen erleben die Schüler/ 
innen die Lehrpersonen positiv (siehe Abschn. 7.2) und auch das Arbeiten miteinander wird generell positiv eingeordnet. Allerdings thematisieren die Schüler/innen auch Konflikte: ,wenn die Lehrperson dann manchmal auch nach unten zu denen die dann am Projekt gearbeitet haben, nach-also dahin gegangen ist, da war halt oben, ham die sich jetzt nicht so viel Mühe gemacht irgendwie zu lernen, dann war- ham die oben eigentlich nur gequatscht oder (.) ja. irgendwas gemacht was sie eigentlich nicht machen sollten“. Das hier thematisierte ,Quatschen" verweist auf Unruhe im Klassenraum, die von einigen Schüler/innen bemängelt wird. Diese scheint phasenweise bzw. situationsbezogen aufzutreten. Hervorgehoben werden von den Schüler/ innen aber gleichermaßen auch Gegenmaßnahmen, etwa die Möglichkeit, den Raum zu verlassen oder Kopfhörer aufzusetzen.

Selbst Aufgaben wählen zu können und für das Lernen Verantwortung zu übernehmen ist für die Lernenden von besonderer Bedeutung. Sie messen dieser Möglichkeit einen hohen Wert zu (siehe Abschn. 7.1) und bewerten daraufhin die Atmosphäre in den Lumosstunden insgesamt auch als positiv. Gleichzeitig erleben andere die Lumosstunden unstrukturiert und chaotisch, wie im Folgenden deutlich wird: „Ich fande die Arbeitsatmosphäre war jetzt nicht so gut weil, es war halt alles immer so durcheinander, und der eine hat das Arbeitsblatt gesucht, der andere das, und wir hatten auch immer [...] so Ordner, wo man die Blätter halt so rausziehen konnte, und dann sich jeder halt noch is noch durch die Klasse gelaufen, andere ham was nicht verstanden, ham dann mit'm Nachbar geredet, also das war nicht so gut“.

\section{Diskussion}

Erkennbar ist auf der konzeptionellen Ebene, dass mit den Lumosstunden die Maxime ,Individuelle Förderung für alle' verfolgt wird. Während die Bildungsadministration die Ergänzungsstunden als kompensatorische Zusatzangebote versteht, die die Möglichkeit der Profilbildung und Stärkenorientierung bieten können, aber nicht müssen, werden die Lumosstunden als ein Angebot für alle Schüler/innen konzipiert. Dabei bleibt der kompensatorische Ansatz stark erhalten, Profilbildungen werden aber ermöglicht und in den späteren Jahrgangsstufen stärker erkennbar (ab Klasse 7). Es werden nicht nur kognitive Dimensionen des Lernens angesprochen, sondern auch soziale Aspekte gefördert (etwa Sozialtraining im Klassenverband). Strukturiert werden die Maßnahmen insgesamt nach dem Prinzip der äußeren Differenzierung vor allem ab der Klasse 7. Zuvor erfolgt die Strukturierung stark an der Ausrichtung am Unterrichtsfach (Wo soll geübt werden?) bzw. über das zur Verfügung stehende Material oder über die Lehrpersonen, die die Lumosstunden begleiten bei einer stärkeren Orientierung an den eigenen Unterrichtsfächern.

Erkennbare Strategie für die konkrete Ausgestaltung individueller Förderung ist in den Lumosstunden eine starke Orientierung an Lern- und Arbeitsstrategien, mit denen darauf abgezielt wird, dass selbstregulierte Lernen zu fördern und die Eigenverantwortlichkeit der Schüler/innen zu stärken. Die Öffnungen der Angebote bieten den Schüler/innen dann ein differenzierteres Angebot, als zunächst in der Konzeption erscheint. Beachtenswert ist, dass hier vor allem die Schüler/innen individualisierte 
Angebote oder Möglichkeiten erhalten, die als leistungsstark zu bezeichnen sind (vor allem ab Klasse 7).

Die inhaltliche Gestaltung der Lumosstunden wird von den Schüler/innen differenziert eingeschätzt. Die Übungsaufgaben werden dann als sinnvoll wahrgenommen, wenn sie anknüpfen an konkret anfallende Probleme oder Fragen bzw. wenn sie direkt auf anstehende Klassenarbeiten vorbereiten. Das Üben in der Schule wird positiv bewertet, da hier Gespräche mit anderen Schüler/innen oder Lehrpersonen helfen können, anfallende Probleme zu beheben.

Problematisiert werden die Aufgaben dann, wenn der vorgegebene zeitliche Rahmen als zu knapp oder zu weit wahrgenommen wird oder wenn der Bedarf zu üben nicht gesehen wird, etwa dann, wenn Inhalte beherrscht werden oder die Notensituation eine sehr gute ist. Die Schüler/innen haben aus der eigenen Perspektive zu wenig Möglichkeiten, Schwerpunkte zu setzen, gezielt Material zu nutzen bzw. auf Übungen zu verzichten. Die Freiheit in der Bearbeitung wird positiv herausgestellt. Mit Blick auf die theoretische Grundlegung individueller Förderung ist die Konzentration auf das Üben als eine deutliche Verknappung zu bewerten: Die Orientierung an den Schwierigkeiten der Schüler/innen ist maßgeblich für die Organisation individueller Förderung. Angebote, die darauf abzielen, vorhandene Stärken oder Interessen auszubauen, sind dagegen in den Klassen 5 und 6 lediglich durch die Projekte gegeben, in denen die Schüler/innen eigene Themen verfolgen. Diese können aber nur von einigen Schüler/innen angegangen werden. Dadurch, dass alle Schüler/innen alle Übungsaufgaben bearbeiten müssen, ist eine Differenzierung nur scheinbar bzw. anteilig gegeben. Durch die Setzung von Schwerpunkten haben die Schüler/innen die Möglichkeit, etwa die Reihenfolge der Arbeitsschritte selbst $\mathrm{zu}$ bestimmen. Autonom in ihrer Entscheidung, welche Aufgaben sie bearbeiten, sind sie aber nicht. Im Sinne der individuellen Förderung als Gesamtunterrichtsstrategie wäre es sinnvoll, den gegebenen Rahmen flexibler zu nutzen und Aufgaben bereitzustellen, die ein differenziertes Anforderungsniveau bereithalten.

Vorausgesetzt wird von den Schüler/innen, dass nicht nur die Aufgaben, sondern auch die Lernumgebung durch die Lehrpersonen gut vorbereitet sind. Fehlende Materialien oder Kontrollbögen werden angemahnt, gut strukturierte Materialien als Gewinn wahrgenommen. Mangelnde Absprachen zwischen den beteiligten Lehrpersonen werden moniert. Gewünscht wird eine Erweiterung dieser Arbeitsweise auf weitere Fächer. Dabei betonen die Schüler/innen, dass die Eigenverantwortung ein zentraler Ansatz für das Gelingen ist. Die Unterstützung der Lehrpersonen bleibt ein wichtiger Gelingensaspekt. Hierin zeigt sich, dass die Schüler/innen wahrnehmen, wann Unterricht aus ihrer Perspektive erfolgreich sein kann, bzw. wann sich fundamentale Probleme ergeben, die das Lernen behindern. Damit wird auch deutlich, dass die Kommunikation zwischen den Lehrpersonen von den Schüler/innen wahrgenommen und beschrieben werden kann: Die Interaktion zwischen den beteiligten Personen erscheint als ein klares Kriterium für die Einschätzungen zur individuellen Förderung.

Die Lumosstunden bieten neben dem Üben Raum für weitere Aktivitäten, die als gewinnbringend eingeschätzt werden. Etwa dann, wenn Schüler/innen unterschiedlicher Grundschulen in der fünften Klasse zusammenkommen. Hier erweisen sich die Lumosstunden als eine Art Schonraum, in dem zentrale Dinge besprochen oder 
eingeübt werden können, die im regulären Unterricht eine besondere Relevanz gewinnen. Die Schüler/innen können aus ihrer Sicht Freiräume nutzen, um mit den Mitschüler/innen zu sprechen und erleben sich als selbstbestimmt. Dies wird auch deutlich, wenn die moderierende bzw. strukturierende Funktion der Lumosstunden angesprochen wird: Sie werden als ein besonderes Angebot im Alltag wahrgenommen und als hilfreich erlebt, um ,den richtigen Unterricht" mit verfolgen zu können oder sich auf Klassenarbeiten vorzubereiten. Wird individuelle Förderung aber als Gesamtunterrichtsstrategie verstanden, zeigt sich, dass die Ergänzungsstunden als ein Zusatz auch zu problematisieren sind. Während im regulären Unterricht das Angebot für alle Schüler/innen gleich ist, bieten die Ergänzungsstunden Möglichkeiten zur individuellen Förderung. Damit wird aber zu kurz gegriffen, da individuelle Förderung eben nicht nur als eine punktuelle Aufgabe erscheint, sondern vielmehr flächendeckend als Konzept angefragt ist.

Die Äußerungen zu den Projekten, die gestaltet werden können, wenn die Übungsaufgaben erledigt sind, verweisen ebenfalls auf die Bedeutung der Selbstbestimmung. Die Wahl der Themen und Produkte wird positiv herausgestellt, allerdings wird das wiederholte Durchlaufen als nicht sinnvoll wahrgenommen. Hier wäre es aus Sicht der Schüler/innen sinnvoll, andere Formate zu finden, in denen sie ihren Interessen nachgehen können bzw. ihre Arbeits- und Lernstrategien weiter verbessern können.

Die Rolle der Lehrpersonen wird im Vergleich zum Regelunterricht als verändert beschrieben. Sie sind die Expert/innen für fachliche Inhalte, nehmen aber verstärkt eine beratende und begleitende Rolle ein. Kontrollierende Funktionen nehmen dagegen ab. Hierdurch ergeben sich auch Reibungen innerhalb der Klassen, was darauf hinweist, dass die offene Arbeitsform eingeübt und begleitet werden muss. Hier lassen sich Rückschlüsse auf die vorbereitete Lernumgebung ziehen: Die Lehrpersonen müssen mit den Schüler/innen Regeln für offenes Unterrichten finden. Die Verantwortung liegt dann nicht nur bei den Lehrpersonen, sondern auch bei den Schüler/ innen, die diese Freiheiten honorieren müssten, um sie nicht zu verlieren. Deutlich wird, dass Konsequenzen nicht durchgängig vereinbart sind. Bemerkenswert ist auch, dass die Schüler/innen diese veränderte Rolle der Lehrpersonen würdigen: Sie erkennen, welche Herausforderungen sich für diese ergeben. Dies erscheint als ein guter Ansatzpunkt zur weiteren Ausgestaltung solcher offenen Formate. Dass geöffneter Unterricht, wie er in den Lumosstunden angeboten wird, von den Schüler/innen positiv wahrgenommen wird, unterstützt diese Annahme. Gerade in dieser Schulform sind geöffnete Formate vergleichsweise selten und bieten Entwicklungsmöglichkeiten, um individuelle Förderung auszugestalten.

\section{Fazit}

Die in dieser Studie gewonnenen Ergebnisse bieten mit Blick auf die Beforschung der individuellen Förderung im Allgemeinen und der Ergänzungsstunden im Speziellen keine verallgemeinerbaren Perspektiven für die Umsetzung und Durchführung von Maßnahmen individueller Förderung. Gezeigt werden kann aber, ausgehend von dieser vertieften Perspektive an einer exemplarisch ausgewählten Schule, dass die 
Sichtweisen der Schüler/innen tatsächlich einen Mehrwert für die wissenschaftliche Betrachtung bringen können.

Es zeigt sich: Schüler/innen sind in der Lage, ihre schulische Situation differenziert zu beschreiben. Mit den Gruppendiskussionen ist zudem eine Form gefunden worden, in der die Schüler/innen ins Gespräch finden, auch wenn mit forschenden Personen fremde Akteur/innen hinzutreten. Die diskursive Auseinandersetzung mit den Lumosstunden verdeutlicht, dass es ein großes Potenzial sowohl für die schulische als auch die wissenschaftliche Praxis ist, Schüler/innen explizit in Forschungsprozesse einzubinden. Gerade durch die Einbindung externer Akteur/innen ist davon auszugehen, dass die Schüler/innen etwa in Evaluationsvorhaben, bereit sind, sich kritischer zu äußern, als dies etwa im Feedback für Lehrpersonen erfolgen kann. Dadurch, dass die Schüler/innen die Lumosstunden in unterschiedlicher Ausprägung bzw. in Vorläufermodellen kennen, ist eine diskursive Auseinandersetzung in den gemischten Gruppendiskussionen sichergestellt. Im Sinne von Entwicklungsprozessen können die Schüler/innen mit Blick auf evaluative Aufgaben dazu beitragen, Angebote zu verbessern, wenn ihre Positionen und Argumentationen von den Verantwortlichen aufgenommen werden. Für die Forschung bieten sie eine spannende Perspektive an: Sie sind diejenigen, die Unterricht erfahren und die hier eingebunden sind. Neben den Perspektiven von Lehrpersonen können die Perspektiven von Schüler/innen also helfen, das Unterrichtsgeschehen detaillierter zu untersuchen.

Die Ergänzungsstunden sind ein weites Feld und bislang noch unterbeforscht. Dieser Beitrag bietet eine Perspektive, wie die Ergänzungsstunden analytisch betrachtet werden können. In Verbindung mit weiteren Quellen, etwa den Lehrpersonen oder den Leistungsentwicklungen der Schüler/innen, lassen sich differenzierte Betrachtungen individueller Förderung erstellen. Dies gilt gerade für die Schulform Gymnasium. Deutlich wird aber auch, dass die Ergänzungsstunden, wenn sie eingesetzt werden wie die Lumosstunden, nur bedingt der Idee individueller Förderung entsprechen. Hier bedarf es differenzierter Analysen bestehender Konzepte. Vor allem dann, wenn individuelle Förderung als gesamtunterrichtliche Strategie zu verstehen ist und nicht nur als ausgelagerte Form der Leistungskompensation.

Deutlich wird, dass Maßnahmen individueller Förderung nicht rein leistungsbezogen relevant sind, sondern darüber hinaus für die Selbstwahrnehmung der Schüler/ innen eine besondere Kraft entwickeln können. Die Möglichkeit, Angebote offen zu gestalten und die Verantwortung der Lernenden in diesem Sinne zu stärken, erscheint aus Sicht der Schüler/innen als wichtiger Ansatzpunkt für die Ausgestaltung entsprechender Maßnahmen. Dies aber erfordert Mut und Offenheit der Schulen als Institution und auch der Lehrpersonen, die solche Formate verantworten. Die Konflikte, die aufgezeigt wurden, verdeutlichen dies in exemplarischer Weise.

Förderung Das Projekt ,Dealing with Diversity` der Westfälischen Wilhelms-Universität Münster wird im Rahmen der gemeinsamen „Qualitätsoffensive Lehrerbildung“ von Bund und Ländern aus Mitteln des Bundesministeriums für Bildung und Forschung gefördert.

Funding Open Access funding enabled and organized by Projekt DEAL.

Open Access Dieser Artikel wird unter der Creative Commons Namensnennung 4.0 International Lizenz veröffentlicht, welche die Nutzung, Vervielfältigung, Bearbeitung, Verbreitung und Wiedergabe in jeglichem Medium und Format erlaubt, sofern Sie den/die ursprünglichen Autor(en) und die Quelle ord- 
nungsgemäß nennen, einen Link zur Creative Commons Lizenz beifügen und angeben, ob Änderungen vorgenommen wurden.

Die in diesem Artikel enthaltenen Bilder und sonstiges Drittmaterial unterliegen ebenfalls der genannten Creative Commons Lizenz, sofern sich aus der Abbildungslegende nichts anderes ergibt. Sofern das betreffende Material nicht unter der genannten Creative Commons Lizenz steht und die betreffende Handlung nicht nach gesetzlichen Vorschriften erlaubt ist, ist für die oben aufgeführten Weiterverwendungen des Materials die Einwilligung des jeweiligen Rechteinhabers einzuholen.

Weitere Details zur Lizenz entnehmen Sie bitte der Lizenzinformation auf http://creativecommons.org/ licenses/by/4.0/deed.de.

\section{Literatur}

Bellmann, J. (2016). Der Aufstieg der Bildungswissenschaften und das sozialtheoretische Defizit der Erziehungswissenschaft. In N. Ricken, R. Casale \& C. Thompson (Hrsg.), Die Sozialität der Individualisierung (S. 51-70). Paderborn: Schöningh.

Bosse, S., Jaeuthe, J., Lambrecht, J., Bogda, K., Koch, H., \& Spörer, N. (2018). Die Sicht von Kindern auf Inklusion in der Schule: Die Entwicklung eines Messinstruments zur Erhebung der Einstellung zum gemeinsamen Lernen im Grundschulalter. Empirische Sonderpädagogik, 4, 329-345.

Corno, L. (2008). On teaching adaptively. Educational Psychologist, 43(3), 161-173. https://doi.org/10. 1080/00461520802178466.

Fischer, C. (2014). Individuelle Förderung als schulische Herausforderung. Bonn: bub.

Flick, U. (2012). Qualitative Sozialforschung. Eine Einführung. Reinbek bei Hamburg: Rowohlt.

Flores, J., \& Alonso, C. (1995). Using focus groups in educational research. Exploring teachers' perspectives on educational change. Evaluation Review, 19(1), 84-101. https://doi.org/10.1177/ 0193841 X9501900104.

van Geel, M., Keuning, T., Frèrejean, J., Dolmans, D., van Merriënboer, J., \& Visscher, A. J. (2019). Capturing the complexity of differentiated instruction. School Effectiveness and School Improvement, 30(1), 51-67. https://doi.org/10.1080/09243453.2018.1539013.

Gläser-Zikuda, M. (2011). Qualitative Auswertungsverfahren. In H. Reinders, H. Ditton, B. Gniewosz \& C. Gräsel (Hrsg.), Empirische Bildungsforschung. Eine Einführung (S. 109-119). Wiesbaden: VS.

Gruehn, S. (2000). Unterricht und schulisches Lernen. Schüler als Quellen der Unterrichtsbeschreibung. Münster: Waxmann.

Hasselhorn, M., Decristan, J., \& Klieme, E. (2018). Individuelle Förderung. In O. Köller, M. Hasselhorn, F. Hesse, K. Maaz, J. Schrader, H. Solga, C. K. Spieß \& K. Zimmer (Hrsg.), Das Bildungswesen in Deutschland. Bestand und Potenziale (S. 375-408). Bad Heilbrunn: Klinkhardt.

Hattie, J. (2014). Lernen sichtbar machen. Baltmannsweiler: Schneider Hohengehren.

Heinzel, F. (2013). Zugänge zur kindlichen Perspektive - Methoden der Kindheitsforschung. In B. Friebertshäuser, A. Langer \& A. Prengel (Hrsg.), Handbuch Qualitative Forschungsmethoden in der Erziehungswissenschaft (S. 707-722). Weinheim: Beltz.

Helmke, A. (2010). Unterrichtsqualität und Lehrerprofessionalität. Diagnose, Evaluation und Verbesserung des Unterrichts. Seelze-Velber: Friedrich-Verlag.

Hess, M., \& Lipowsky, F. (2017). Lernen individualisieren und Unterrichtsqualität verbessern. In F. Heinzel \& K. Koch (Hrsg.), Individualisierung im Grundschulunterricht. Anspruch, Realisierung und Risiken (S. 23-31). Berlin, Heidelberg, New York: Springer.

Klieme, E., \& Warwas, J. (2011). Konzepte der Individuellen Förderung. Zeitschrift für Pädagogik, 57(6), $805-818$.

Kuckartz, U. (2016). Qualitative Inhaltsanalyse - Methoden, Praxis, Computerunterstützung. Weinheim: Beltz.

Kühn, S. (2014). Gymnasiale Strukturreformen und individuelle Förderung: Routine oder Veränderungsimpuls? Eine explorative empirische Analyse. Die deutsche Schule, 106(2), 119-140.

Kunze, I. (2010). Begründungen und Problembereiche individueller Förderung in der Schule - Vorüberlegungen zu einer empirischen Untersuchung. In I. Kunze \& C. Solzbacher (Hrsg.), Individuelle Förderung in der Sekundarstufe I und II (S. 13-26). Baltmannsweiler: Schneider Hohengehren.

Lindemann, S., Glesemann, B., \& Jäger, D. (2014). Individuelle Förderung als Entwicklungsaufgabe für Ganztagsgymnasien. Die Deutsche Schule, 106(2), 141-149. 
Lingemann, J. (2017). Individuelle Förderung am Gymnasium. Eine schultheoretische Mehrebenenperspektive auf die spezifischen Rezeptions-und Anpassungsprozesse in Niedersachsen. https:// repositorium.ub.uni-osnabrueck.de/bitstream/urn:nbn:de:gbv:700-2018020916624/7/thesis_lingema nn.pdf. Zugegriffen: 7. Dez. 2020.

Mäder, S. (2013). Die Gruppendiskussion als Evaluationsmethode - Entwicklungsgeschichte, Potenziale und Formen. Zeitschrift für Evaluation, 12(1), 23-51.

Nunner-Winkler, G. (1971). Chancengleichheit und individuelle Förderung. Eine Analyse der Ziele und Konsequenzen moderner Bildungspolitik. Stuttgart: Enke.

Obrusnikova, I., Block, M., \& Dillon, S. (2010). Children's beliefs toward cooperative playing with peers with disabilities in physical education. Adapted Physical Activity Quarterly, 27(2), 127-142.

Rott, D. (2017). Die Entwicklung der Handlungskompetenz von Lehramtsstudierenden in der Individuellen Begabungsförderung. Forschendes Lernen aufgezeigt am Forder-Förder-Projekt Advanced. Münster: Waxmann.

Schulministerium Nordrhein-Westfalen (o.J.). Weiterentwicklung des Gymnasiums (G8/G9). https://www. schulministerium.nrw.de/docs/Schulpolitik/G8-G9/index.html. Zugegriffen: 7. Dez. 2020.

Solzbacher, C. (2016). Positionen von Lehrerinnen und Lehrern zur individuellen Förderung in der Sekundarstufe I -Ergebnisse einer empirischen Untersuchung. In I. Kunze \& C. Solzbacher (Hrsg.), Individuelle Förderung in der Sekundarstufe I und II (S. 33-53). Baltmannsweiler: Schneider Hohengehren.

Trautmann, M., \& Wischer, B. (2011). Heterogenität in der Schule. Eine Einführung. Berlin, Heidelberg, New York: Springer. 